\title{
Comparison of Integrated Radiation Transport Models with TEPC Measurements for the Average Quality Factors in Spaceflights
}

\author{
Myung-Hee Y. Kim ${ }^{1}$, Hooshang Nikjoo ${ }^{2}$, John F. Dicello ${ }^{3}$, Vincent Pisacane ${ }^{3}$, \\ and Francis A. Cucinotta ${ }^{4}$ \\ ${ }^{1}$ Wyle Laboratories, Inc., Houston, TX 77058 \\ ${ }^{2}$ USRA Division of Space Life Sciences, Houston, TX 77058 \\ ${ }^{3}$ US Naval Academy, Annapolis, MD 21402 \\ ${ }^{4}$ NASA Lyndon B. Johnson Space Center, Houston, TX 77058
}

The purpose of this work is to test our theoretical model for the interpretation of radiation data measured in space. During the space missions astronauts are exposed to the complex field of radiation type and kinetic energies from galactic cosmic rays (GCR), trapped protons, and sometimes solar particle events (SPEs). The tissue equivalent proportional counter (TEPC) is a simple time-dependent approach for radiation monitoring for astronauts on board the International Space Station. Another and a newer approach to Microdosimetry is the use of silicon-on-insulator (SOI) technology launched on the MidSTAR-1 mission in low Earth orbit (LEO). In the radiation protection practice, the average quality factor of a radiation field is defined as a function of linear energy transfer (LET), Qave $(L E T)$. However, TEPC measures the average quality factor as a function of the lineal energy $y, Q_{a v e}(y)$, defined as the average energy deposition in a volume divided by the average chord length of the volume. Lineal energy, $y$, deviates from $L E T$ due to energy straggling, delta-ray escape or entry, and nuclear fragments produced in the detector volume.

Monte Carlo track structure simulation was employed to obtain the response of a TEPC irradiated with charged particle for an equivalent site diameter of $1 \mu \mathrm{m}$ of wall-less counter. The calculated data of the energy absorption in the wall-less counter were compiled for various $y$ values for several ion types at various discrete projectile energy levels. For the simulation of TEPC response from the mixed radiation environments inside a spacecraft, such as, Space Shuttle and International Space Station, the complete microdosimetric TEPC response, $f(y, E, Z$ ), were calculated with the Monte Carlo theoretical results by using the first order Lagrangian interpolation for a monovariate function at a given $y$ value $(y=0.1 \mathrm{keV} / \mu \mathrm{m}-5000 \mathrm{keV} / \mu \mathrm{m})$ at any projectile energy level ( $E=0.01 \mathrm{MeV} / \mathrm{u}$ to $50,000 \mathrm{MeV} / \mathrm{u}$ ) of each specific radiation type ( $Z=1$ to 28 ).

Because the anomalous response has been observed at large event sizes in the experiment due to the escape of energy out of sensitive volume by delta-rays and the entry of deltarays from the high-density wall into the low-density gas-volume cavity, Monte Carlo simulation was also made for the response of a walled-TEPC with wall thickness $2 \mathrm{~mm}$ and density $1 \mathrm{~g} / \mathrm{cm}^{3}$. The radius of cavity was set to $6.35 \mathrm{~mm}$ and a gas density $7.874 \times 10^{-5} \mathrm{~g} / \mathrm{cm}^{3}$. The response of the walled- and the wall-less counters were compared.

The average quality factor $Q_{a v e}(y)$ for trapped protons on STS-89 demonstrated the good agreement between the model calculations and flight TEPC data as shown in figure 1. 
Using an integrated space radiation model (this includes the transport codes HZETRN and BRYNTRN, the quantum nuclear interaction model QMSFRG) and the resultant response distribution functions of walled-TEPC from Monte-Carlo track simulations, we compared model calculations with walled-TEPC measurements from NASA missions in LEO and made predictions for the lunar and the Mars missions. The $Q_{a v e}(y)$ values for the trapped or the solar protons ranged from 1.9-2.5. This over-estimates the $Q_{a v e}(L E T)$ values which ranged from 1.4-1.6. Both quantities increase with shield thickness due to nuclear fragmentation. The $Q_{a v e}(L E T)$ for the complete GCR spectra was found to be 3.5-4.5, while flight TEPCs measured 2.9-3.4 for $Q_{a v e}(y)$. The GCR values are decreasing with the shield thickness. Our analysis for a proper interpretation of data supports the use of TEPCs for monitoring space radiation environment.

\section{ACKNOWLEDGEMENTS}

This work was supported by the National Space Biomedical Research Institute through NASA NCC 9-58.

STS-89

Trapped Radiation

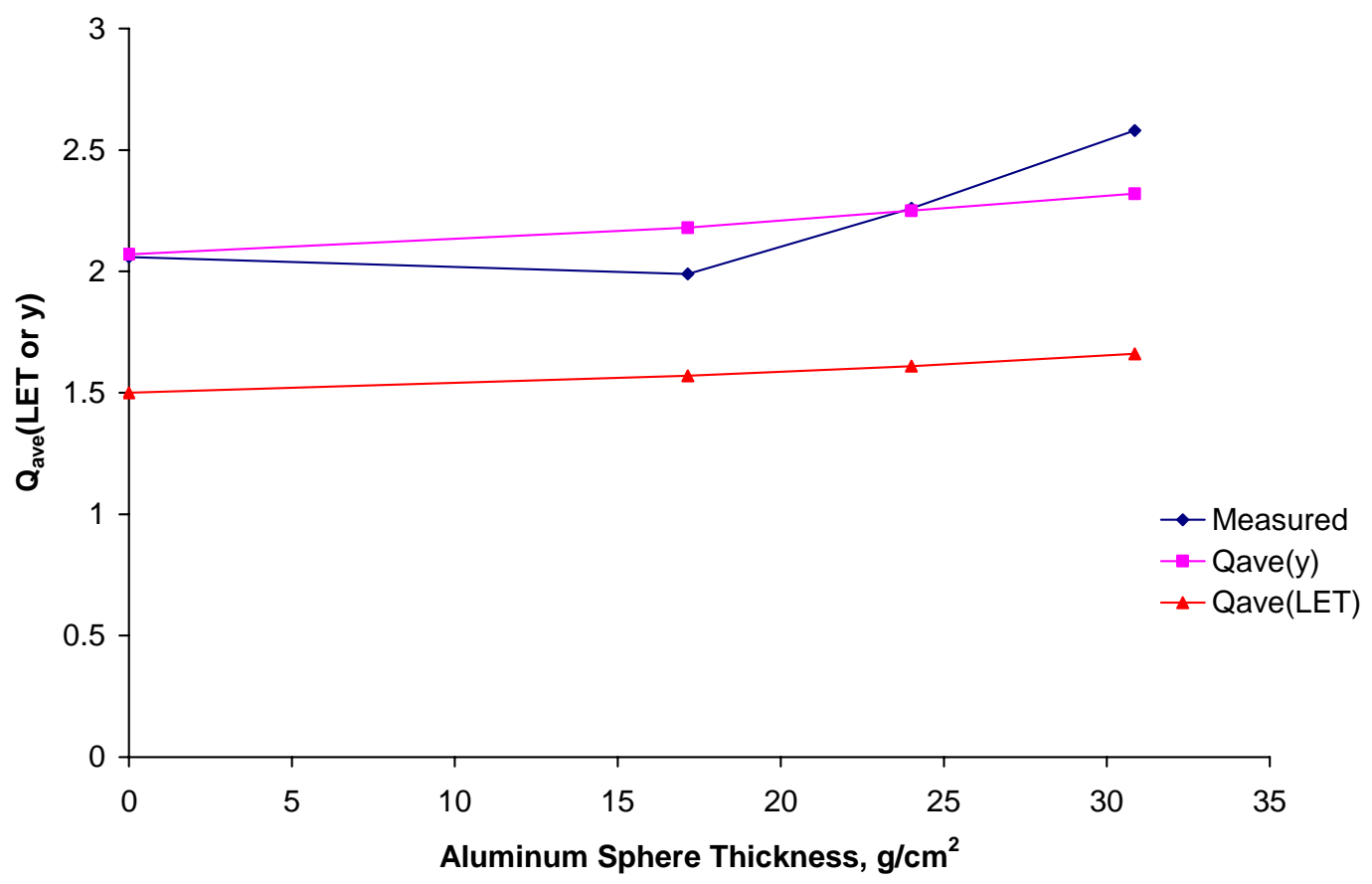

Figure 1. Average quality factor for trapped protons as a function of lineal energy y or LET by model calculations and the TEPC measurements from the STS-89 mission. 